\title{
Identidad nacional como punto de encuentro y flexibilización académica: la utilización de universalidades mexicanas y especificidades estatales-municipales para la creación de un sistema educativo media superior de calidad
}

Fabián Hernández Galicia, Universidad Autónoma del Estado de Hidalgo y Colegio de Bachilleres del Estado de Hidalgo, México

\begin{abstract}
Resumen: En México se está viviendo una época de reestructuración legal, especialmente en materia educativa, las medidas legislativas han propiciado un ambiente social y laboral optimista. En este marco, los grupos sociales involucrados en la Educación Media Superior deben crear protocolos de acción institucionales que promuevan una identidad nacional, estatal y municipal, con las que se interioricen significados sociales de tolerancia, inclusión, tranquilidad y paz. El presente proyecto demuestra, a manera de ejercicio en el Colegio de Bachilleres del Estado de Hidalgo, la creación de ejes fundamentales a través de las prácticas académicas cotidianas.
\end{abstract}

Palabras clave: identidad, nacional, educación, sociedad, calidad, inclusión, tolerancia, municipal, estatal, cotidianeidad, flexibilización

\begin{abstract}
In Mexico it is experiencing a time of legal restructuring, especially in education, legislative measures have led to an optimistic social and work environment. In this context, the social groups involved in education must create institutional protocols of action that promote a national and municipal identity that promotes the internalize social meanings of tolerance, inclusion, and peace. This project became from collaborate working in the Colegio de Bachilleres del Estado de Hidalgo.
\end{abstract}

Keywords: Identity, Education, Society, Quality, Inclusión, Tolerance, Municipal, Easing

\section{Introducción}

$\mathrm{E}$ n la actualidad, se pretende desde diversos ámbitos sociales y culturales que la educación adquiera características transversales de calidad, eficacia, eficiencia e inclusión social. En el último tramo gubernamental de México, estas preocupaciones han sido plasmadas en las reformas estructurales de la nación. En especial, la Reforma Educativa le brinda carácter de legalidad a los nuevos requerimientos sociales. ${ }^{1}$

La sociedad ha expresado de diversas maneras, causas y consecuencias, la necesidad por una Reforma Educativa en los ámbitos nacionales, estatales y municipales. Según el Gobierno de México, este proceso de actualización tiene ejes de acción definidos:

La educación pública es laica y gratuita, y obligatoria la de los tipos básico y medio superior.

La Reforma Educativa se realiza para que la educación sea de calidad, otorgue igualdad de oportunidades a los mexicanos y garantice el interés superior de la niñez.

Con la Reforma Educativa la Educación será incluyente, distribuyendo mayores recursos a las zonas más pobres de México.

La Reforma Educativa se propone fortalecer el papel de la educación para reducir las desigualdades sociales.

\footnotetext{
${ }^{1}$ SEV. Recuperado de http://www.sev.gob.mx/educacion-media-superior-y-superior/files/2013/09/Reformas-
}

Revista Internacional de Educación y Aprendizaje

Volumen 3, Número $2<\mathrm{http}$ ://sobrelaeducacion.com/>, ISSN 2255-453X

(C) Global Knowledge Academics. Fabián Hernández Galicia

Todos los derechos reservados. Permisos: soporte@gkacademics.com 
Se mejorarán las condiciones físicas, materiales y pedagógicas en las escuelas.

Nada debe entorpecer la vida normal de las escuelas y el aprendizaje de los alumnos.

Todos los sectores — público, social y privado — deben participar activamente para que la educación en México sea de mayor calidad y se brinde a todos los niños y jóvenes. ${ }^{2}$

Como se puede observar, la educación ha sufrido un cambio sustancial, social y de mayor forma, legal. Estos cambios permiten utilizar nuevas estrategias educativas, así mismo proponer alternativas de solución académica con matices sociales y culturales.

El propósito de la presente ponencia, es presentar alternativas educativas, esencialmente en los Sistemas de Educación Media Superior, que promuevan una congruencia entre los ejes de las Reforma Educativa, las nuevas necesidades de la sociedad moderna o líquida (Bauman 2007:111) y los fines de la Educación Media Superior (EMS).

Presentando un marco teórico referencial que justifique un punto de encuentro entre categorías de análisis culturales y alternativas de solución mediante la utilización transversal de los sistemas de EMS. La recuperación de temas centrales de la Reforma Integral Media Superior (RIEMS) como el modelo por competencias y situaciones inherentes a él, así como la integración del pensamiento posmoderno o de crítica a la modernidad ${ }^{3}$ como matriz de resignificación (Casas:20:2007) de sistema de valores necesario para la creación de una identidad como alternativa de solución planteada por Castells (321:1995):

...conexión común con la comunicación universal a las redes de comunicación mundiales y a un inmenso circuito de intercambios, abierto a recibir mensajes y experiencias que abarcan el mundo entero. En el otro extremo, las redes locales fragmentadas con frecuencia definidas étnicamente, utilizan su identidad como el recurso más precioso para defender sus intereses y hasta su propia existencia.

Para continuar con el alineamiento argumentativo del presente escrito, se detalla la metodología de acción para la implementación de la propuesta de intervención y se presenta un bosquejo general, basado en ejemplos concretos. En esta parte, se pretende dejar clara la relación entre la glocalización ${ }^{4}$ educativa y la resignificación del sistema de valores como detonantes de la identidad nacional, estatal y municipal.

Al finalizar, se presentan conclusiones plenarias, en las que se expresan las inquietudes detectadas y congruencias que han surgido mediante la práctica docente, y a su vez, se clarifican los resultados esperados. Asimismo se establecen limites, limitantes y fortalezas de la proyección y puesta en marcha de la propuesta de intervención planteada a lo largo del escrito.

\section{Marco teórico}

Las nuevas necesidades de una sociedad líquida, una esfera moderna que propician estructuras efímeras en niveles globales, nacionales y/o locales, nos arrojan a la difícil tarea de crear estructuras formales o institucionales que permitan fortalecer "imaginarios sociales" (Castoriadis:1998:46-47) como base de una "identidad" nacional, estatal y municipal. En otras palabras, el mundo social, como imaginario colectivo, ha creado diversas maneras de ver el contexto, interiorizarlo y reproducirlo.

Diversificar visiones y entendimientos provoca que las estructuras de valores o el sistema de valores, como lo llama Bauman (1999) en su libro "cultura como praxis. En el que podemos observar la naturaleza de la heterogeneidad de valores en la modernidad.

Nuestra época, la época del pluralismo cultural, opuesto a la pluralidad de las culturas, no es un tiempo de nihilismo. Lo que hace la situación humana confusa y las elecciones difíciles no es la ausencia

\footnotetext{
${ }^{2}$ Presidencia de la República. Recuperado de http://www.presidencia.gob.mx/reformaeducativa/ el 28 de febrero de 2014.

${ }^{3}$ Rama del pensamiento sociológico contemporáneo basado en la crítica racional a la sociedad del consumo y la manera en la que permea inconscientemente en el accionar de todos los grupos sociales occidentales.

4 "Nuestra identidad no puede definirse ya por la pertenencia exclusiva a una comunidad nacional. El objeto de estudio no debe de ser entonces sólo la diferencia, sino también la hibridación" (Beck 1998:79).
} 
de valores o la pérdida de su autoridad, sino la multitud de valores, escasamente coordinados y débilmente vincula- dos a toda una discordante variedad de autoridades.

Asimismo, las repercusiones de la multiplicidad de valores se están viviendo en los contextos escolares, como productos de un contexto social ineludible. Es de preocupación del sistema educativo encontrarse con problemáticas tan complejas y difíciles de detectar. Sin embargo, es aún más importante para la educación institucional en México, encontrar una solución bien definida y con promesas de funcionamiento, alcance y temporalidad razonable.

Se han creado diversas políticas públicas en materia educativa, complejos currículos de educación básica y Media Superior, y los más importantes para este escrito: los espacios de reunión académica. Los Foros de Consulta Nacional para la Revisión del Modelo Educativo son espacios de diálogo especializado y que promueven soluciones, previsiones y ajustes a los procesos educativos nacionales. Lo anterior es de vital importancia, ya que por medio de estos Foros, se pretende crear la discusión detonante para la creación curricular o remodelación del currículo con inclusión de términos propuestos por esta ponencia. En resumen, tener estos espacios de diálogo promoverá "universalidades" nacionales y "especificidades" locales con un alineamiento que converja en la "identidad nacional". Promover la identidad, como lo analiza Castells, es resignificar el sistema de valores (múltiple en la actualidad) para un rescate de la idea nacional.

Por otra parte, es necesario explicar el término universalidad, ya que es la vertiente cultural por la que se desplaza la hipótesis sobre homogeneidad del sistema de valores. Las universalidades, de manera epistemológica, aluden a las características que se encuentran en diversos actores sociales, sin importar la ubicación geográfica o su grado de cohesión social. Por ejemplo, Una universalidad mexicana, en la década de los noventa, era la preocupación por la creciente y acaparadora globalización así como sus efectos económicos, culturales y sociales.

El concepto de universalidad brinda herramientas de creación de identidad, utilizando el espacio y recursos humanos-materiales de los subsistemas de EMS. Es decir, creando universalidades mexicanas consensuadas se promueve la creación de varios puntos de encuentro de identidad. Aprovechando la circunstancia de homogeneización empírico cultural, se propone guiar la construcción de universalidades hacia una resignificación del sistema de valores. Lo inmediato anterior, siempre y cuando se promueva la creación de una estructura que funcione como promotora de una cohesión social y ésta, a su vez, sea una propuesta de intervención, a lo que mencionamos con preocupación, el sistema de valores con características de fluidez.

En resumen, la creación de universalidades será promotora de un cambio paulatino en la creación de identidad. No sólo tener a los símbolos patrios como proyecciones de identidad, sino crear por lo menos dos universalidades que permeen los grupos sociales anexados al sistema mexicano.

Asimismo, la creación de una identidad nacional no debe descuidar la reciprocidad entre lo global y lo local. Es decir, tener en cuenta que México, como país, debe respetar y procurar las particularidades de cada espacio geográfico- social perteneciente al sistema de gobierno. Por consecuencia de lo anterior, es prioridad crear de manera inherente a las universalidades, características educativas locales, con tendencias específicas. El concepto universal, ligado con lo específico, se debe analizar desde la perspectiva de la unidad, como nos menciona Agustín de la Herrán ${ }^{5}$ :

Podemos entender por universalidad y unidad dos facetas del mismo fenómeno: la universalidad, como sentimiento, actitud, conocimiento, estado de conciencia o mundo interior no fragmentado del sujeto. Y la unidad, como la culminación correspondiente a esa percepción global. En efecto: "La unidad, en la vida humana, no constituye un medio para el logro de ciertos fines. Es el medio por el cual llega el hombre a plenitud. La unidad es una meta.

\footnotetext{
${ }^{5}$ Profesor titular de la Universidad Autónoma de Madrid. Recuperado de http://www.tendencias21.net/La-universalidad-unreferente-para-la-construccion social_a11183.html?utm_source=feedburner\&utm_medium=feed\&utm_campaign= Feed $\% 3 \mathrm{~A}+$ tendencias $21 \% 2 \mathrm{FfPZM}+\overline{\%} 28 \mathrm{~T}$ endencias $+2 \overline{1} \% 29$ el 3 de marzo de $20 \overline{14}$.
} 
En congruencia con lo anterior, especificidades son categorías de análisis sociológico, que aluden a las particularidades de reproducción cultural en un determinado espacio, ya sea geográfico o comunicativo. La importancia de este concepto, recae en la multiplicidad de subculturas mexicanas.

El problema de la identidad es la dualidad que promueve. Por ejemplo, es un proyecto de unidad nacional, pero también de separación local. Procura símbolos nacionales, pero descuida las necesidades locales. Estandariza ideales, empero de la misma forma, crea choques culturales.

Ligando conceptos, las especificidades deben alinear objetivos e ideales, para glocalizar (ver nota al pie 4) la identidad nacional, se debe crear un nodo estratégico entre los objetivos de las universalidades y procurar las generalidades similares en las especificidades locales. El alineamiento metodológico debe ser debatido y consensuado, para esto es importante comprender a los Foros de Consulta Nacional para la Revisión del Modelo Educativo, como las reuniones académicas nacionales y locales de creación de universalidades y especificidades. Proyecto con miras de solidificación del sistema de valores nacional y, a su vez, promotor de la cohesión social.

\section{Metodología de la acción}

En este penúltimo apartado, se pretende ejemplificar el modo accionar del presente proyecto. Entendiendo que la Educación Media Superior ha adquirido el carácter de obligatoriedad, otorgado por la Constitución Mexicana, y por ende el presupuesto e importancia social han ido al alza. Se utiliza esta plataforma educativa, como estructura institucional de formación de universalidades y especificidades en los diferentes aspectos geográfico-sociales.

Como primer paso, utilizar los Foros de Consulta Nacional para la Revisión del Modelo Educativo como reunión académica de creación, debate y concordancia. Es aquí donde se discutirán los ejes nacionales, estatales y municipales para lograr una alineación ideológica, ya que se contará con la presencia de representativos regionales (docentes y directivos) que pueden proponer ejes de acción específicos al mismo tiempo que se generan las universalidades que recubrirán el Marco Curricular Común ${ }^{6}$.

Para la puesta en marcha de la propuesta, ya reunidos en plenaria, se deben debatir las principales necesidades, conflictos, problemáticas sociales, alternativas, soluciones, realidades e imaginarios mexicanos. Asimismo, generar un bosquejo general del entorno nacional, para delimitar y encontrar las universalidades ideales para el desarrollo del proyecto. Esta etapa es el eje fundamental del desarrollo.

Después de generar las universalidades, recordemos que se proponen en la introducción, por lo menos dos de ellas, se discute la alineación con las perspectivas locales (estados y municipios). Recordemos que los Foros se desarrollan por etapas y zonas geográficas cercanas. Asimismo se debe utilizar el entorno para discutir, desde el punto de vista local (docentes y directivos) la misma generación de las especificidades. Así evitar problemas de investigación recurrentes, como el etnocentrismo 7 o tergiversación institucional.

En modo de plenaria, en se deben generar matrices donde se contengan los temas, que debidamente justificados, se consideren universalidades o especificidades. Por ejemplo:

Tabla 1.1: Ejemplo de matriz

\begin{tabular}{|c|c|l|l|}
\hline Concepto & Clasificación & \multicolumn{1}{|c|}{ Justificación social } & \multicolumn{1}{c|}{ Marco Teórico } \\
\hline & & $\begin{array}{l}\text { La situación ambiental ha sido } \\
\text { materia de preocupación mun- } \\
\text { dial, el rescate de los ecosiste- } \\
\text { Preocupación } \\
\text { ambiental. }\end{array}$ & $\begin{array}{l}\text { Universalidad y la conservación de los } \\
\text { recursos naturales debe plan- } \\
\text { tearse como un estilo de vida. } \\
\text { medioambiental, cada día más } \\
\text { patente, y sus consecuencias, } \\
\text { convierte la investigación sobre } \\
\text { las actitudes proambientales y el } \\
\text { comportamiento ecológico en } \\
\text { una cuestión ineludible desde la } \\
\text { perspectiva de la psicología } \\
\text { ambiental." Amerigo (2006). }\end{array}$ \\
\hline
\end{tabular}

\footnotetext{
6 Acuerdo 444, recuperado de http://transparencia.info.jalisco.gob.mx/sites/default/files/ACUERDO\%20444.pdf el 3 de marzo de 2014.
} 
HERNÁNDEZ: IDENTIDAD NACIONAL COMO PUNTO...

\begin{tabular}{|c|c|c|c|}
\hline Concepto & Clasificación & Justificación social & Marco Teórico \\
\hline & & & $\begin{array}{l}\text { “...la idea del Nuevo Paradigma } \\
\text { Ambiental representa un conjun- } \\
\text { to de creencias y valores que se } \\
\text { refieren principalmente a la } \\
\text { valoración de la naturaleza, la } \\
\text { compasión por las otras personas, } \\
\text { la limitación del crecimiento } \\
\text { económico y demográfico, la } \\
\text { evitación de riesgos tecnológicos } \\
\text { y, en general, la participación, } \\
\text { cooperación, el posmaterialismo } \\
\text { y los estímulos de vida sencillos. } \\
\text { El Nuevo Paradigma Medioam- } \\
\text { biental definiría una nueva forma } \\
\text { de organizar y concebir las rela- } \\
\text { ciones sociales que gradualmente } \\
\text { puede incorporar sus concepcio- } \\
\text { nes al sistema social dominante." } \\
\text { González (1999:14). }\end{array}$ \\
\hline $\begin{array}{l}\text { Resignificación } \\
\text { del Sistema de } \\
\text { Valores }\end{array}$ & Universalidad & $\begin{array}{l}\text { La preocupación por una socie- } \\
\text { dad con valores diversos o } \\
\text { líquidos, es de carácter nacional. } \\
\text { Establecer el periodo de identi- } \\
\text { dad, como ya lo mencionamos, } \\
\text { es tarea educativa. }\end{array}$ & $\begin{array}{c}\text { Marco Teórico del presente } \\
\text { escrito. }\end{array}$ \\
\hline $\begin{array}{l}\text { Nuevas } \\
\text { ciudadanías }\end{array}$ & $\begin{array}{l}\text { Especificidad } \\
\text { Estatal. El caso } \\
\text { de Hidalgo. }\end{array}$ & $\begin{array}{l}\text { Dentro de los aspectos fundamen- } \\
\text { tales del sistema de gobierno, se } \\
\text { encuentra la construcción demo- } \\
\text { crática, el poder del pueblo. En } \\
\text { Hidalgo, la participación política } \\
\text { va en aumento, sin embargo, tiene } \\
\text { sus limitantes en tanto a informa- } \\
\text { ción y congruencia. De tal mane- } \\
\text { ra, se considera importante reto- } \\
\text { mar en el ambiente educativo, la } \\
\text { urgente necesidad de crear una } \\
\text { ciudadanía crítica. (Inmediata, ya } \\
\text { que los alumnos de EMS están } \\
\text { cerca de la edad y requerimientos } \\
\text { para adquirir la ciudadanía. }\end{array}$ & $\begin{array}{l}\text { "la nueva ciudadanía tiene la } \\
\text { idea de que las escuelas deben } \\
\text { enseñar a los alumnos a ser } \\
\text { escépticos a propósito de la } \\
\text { autoridad política y a distanciar- } \\
\text { se de sus propias tradiciones al } \\
\text { embarcarse en una discusión } \\
\text { pública no está fuera de contro- } \\
\text { versia. Los tradicionalistas la } \\
\text { rechazan argumentando que esta } \\
\text { política conduce inevitablemen- } \\
\text { te a que también cuestionen la } \\
\text { vida privada, la tradición y la } \\
\text { autoridad paterna o religiosa. Y } \\
\text { esto es seguramente correcto. } \\
\text { Como lo admite Gutmann, la } \\
\text { educación para la ciudadanía } \\
\text { democrática implica necesaria- } \\
\text { mente "dotar a los muchachos } \\
\text { de las habilidades intelectuales } \\
\text { requeridas para evaluar formas } \\
\text { de vida diferentes de la de sus } \\
\text { padres", ya que 'muchas, si no } \\
\text { todas, las capacidades necesarias } \\
\text { para poder elegir entre modelos } \\
\text { de la vida buena son también } \\
\text { necesarias para poder elegir } \\
\text { entre modelos de la sociedad } \\
\text { buena" (Gutmann, 1987:30-40)" }\end{array}$ \\
\hline Pobreza & $\begin{array}{l}\text { Especificidad } \\
\text { Estatal. El caso }\end{array}$ & $\begin{array}{l}\text { Se sobre entiende que el go- } \\
\text { bierno ha implementado accio- }\end{array}$ & $\begin{array}{l}\text { "El término de pobreza tiene, en } \\
\text { general, la connotación de una }\end{array}$ \\
\hline
\end{tabular}




\begin{tabular}{|c|c|c|c|}
\hline Concepto & Clasificación & Justificación social & Marco Teórico \\
\hline & de Hidalgo. & $\begin{array}{l}\text { nes transversales y una de las } \\
\text { principales, el combate contra la } \\
\text { pobreza. Medidas como la Cru- } \\
\text { zada Nacional Contra el Ham- } \\
\text { bre. Bien podría contarse como } \\
\text { una universalidad. En Hidalgo es } \\
\text { necesario redimensionar este } \\
\text { problemas social y generar, junto } \\
\text { con la resignificación del sistema } \\
\text { de valores, una empatía social. }\end{array}$ & 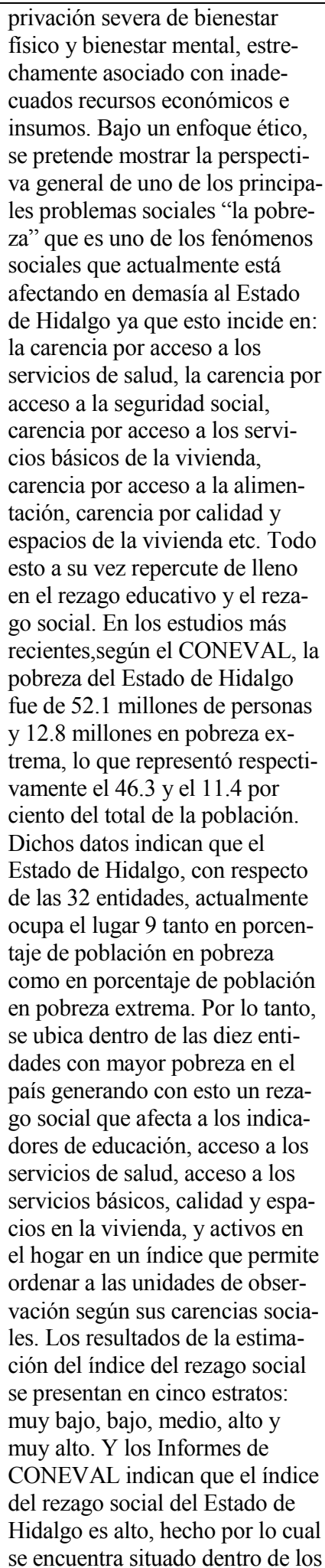 \\
\hline
\end{tabular}


HERNÁNDEZ: IDENTIDAD NACIONAL COMO PUNTO...

\begin{tabular}{|c|c|c|c|}
\hline Concepto & Clasificación & Justificación social & Marco Teórico \\
\hline & & & $\begin{array}{l}\text { primeros } 10 \text { Estados con mayor } \\
\text { índice de rezago social, posicio- } \\
\text { nado en el lugar } 7 .\end{array}$ \\
\hline $\begin{array}{l}\text { Migración y } \\
\text { nuevos } \\
\text { asentamientos } \\
\text { urbanos. }\end{array}$ & $\begin{array}{l}\text { Especificidad } \\
\text { Municipal. El } \\
\text { caso de Tiza- } \\
\text { yuca, Hidalgo. }\end{array}$ & $\begin{array}{l}\text { En el caso de Tizayuca, la crea- } \\
\text { ción de nuevos fraccionamientos } \\
\text { ha llevado a un choque cultural, } \\
\text { falta de recursos naturales y } \\
\text { humanos que garanticen las } \\
\text { necesidades básicas y sobre todo } \\
\text { un desinteré social por el tema. } \\
\text { Comprender un fenómeno social } \\
\text { nos brinda herramientas cogniti- } \\
\text { vas para poder tolerarlo y en } \\
\text { ocasiones, proponer soluciones. }\end{array}$ & $\begin{array}{l}\text { "El rompimiento de los esquemas } \\
\text { agrícolas tradicionales por nue- } \\
\text { vos cultivos comerciales trae } \\
\text { consigo un cambio de la tenencia } \\
\text { de la tierra de usos de explota- } \\
\text { ción basados en el usufructo y la } \\
\text { herencia a cultivos de alta capita- } \\
\text { lización. En esta transformación } \\
\text { de la agricultura las economías } \\
\text { regionales suponen una especia- } \\
\text { lización productiva que esconde a } \\
\text { los mercados mundiales, más que } \\
\text { a los locales, así sucede consecu- } \\
\text { tivamente con otros sectores } \\
\text { primarios de la economía que se } \\
\text { integran al mercado mundial con } \\
\text { una especialización que responde } \\
\text { a la de manda que presentan las } \\
\text { economías industrializadas." } \\
\text { Castells (2000) "El Consejo } \\
\text { Nacional de Población (CONA- } \\
\text { PO) estima que en la actualidad } \\
\text { cerca de } 4 \text { millones de hogares } \\
\text { mexicanos (alrededor de } 18 \text { por } \\
\text { ciento del total) tenían familiares } \\
\text { directos en Estados Unidos, } \\
\text { miembros con antecedentes } \\
\text { migratorios en Estados Unidos o } \\
\text { bien recibían remesas de ese país. } \\
\text { Esta proporción varía amplia- } \\
\text { mente según la región de resi- } \\
\text { dencia. En las entidades que } \\
\text { forman la región tradicional de } \\
\text { emigración, la proporción se } \\
\text { eleva a } 37 \text { por ciento, en el norte } \\
\text { a } 22 \text { por ciento, en el centro a } 12 \\
\text { por ciento y en el sureste a } 4 \text { por } \\
\text { ciento. Estos datos confirman que } \\
\text { el fenómeno migratorio no cons- } \\
\text { tituye un evento aislado o } \\
\text { efimero en la vida de las familias } \\
\text { mexicanas. Por el contrario, se } \\
\text { hace presente a través de la } \\
\text { migración permanente o median- } \\
\text { te el ir y venir de alguno (o algu- } \\
\text { nos) de sus miembros a Estados } \\
\text { Unidos durante muchos años." } \\
\text { Tuirán (2002:79). "El estado } \\
\text { ocupa el quinto lugar de margi- } \\
\text { nación a nivel nacional debido, } \\
\text { entre otras cosas, a que el grueso } \\
\text { de la población se encuentra } \\
\text { desempleada, a que los que }\end{array}$ \\
\hline
\end{tabular}




\begin{tabular}{|c|c|c|c|}
\hline Concepto & Clasificación & Justificación social & Marco Teórico \\
\hline & & & $\begin{array}{l}\text { cuentan con un empleo reciben } \\
\text { bajos salarios y, en términos } \\
\text { generales, a que el estado no } \\
\text { dispone de la infraestructura } \\
\text { necesaria para promover un } \\
\text { desarrollo adecuado. Esto ha } \\
\text { ocasionado que los hidalguenses } \\
\text { que solían emigrar hacia otros } \\
\text { estados del país en busca de } \\
\text { mejores condiciones cambien su } \\
\text { lugar de destino hacia Estados } \\
\text { Unidos, ocupando en } 2003 \text { el } \\
\text { décimo lugar en recepción de } \\
\text { remesas a nivel nacional y el } \\
\text { décimo segundo en expulsión } \\
\text { migratoria con datos de } 2000 \text {. La } \\
\text { región del valle del Mezquital -al } \\
\text { occidente del estado- es la prin- } \\
\text { cipal expulsora de emigrantes al } \\
\text { aportar el } 48.6 \text { por ciento del total } \\
\text { de los migrantes hidalguenses; en } \\
\text { segundo lugar se encuentra la } \\
\text { zona de la Sierra Gorda - ubicada } \\
\text { en los límites con Querétaro- con } \\
14.3 \text { por ciento." Nina Frías } \\
\text { (2006:180) }\end{array}$ \\
\hline $\begin{array}{l}\text { Uso de los Pro- } \\
\text { yectos Integra- } \\
\text { dores como nodo } \\
\text { empírico de la } \\
\text { identidad nacio- } \\
\text { nal. }\end{array}$ & $\begin{array}{l}\text { Especificidad } \\
\text { Municipal. El } \\
\text { caso de Tiza- } \\
\text { yuca, Hidalgo. }\end{array}$ & $\begin{array}{l}\text { Desde el año } 2010 \text { el colegio de } \\
\text { Bachilleres del Estado de Hi- } \\
\text { dalgo implementó en todos y } \\
\text { cada uno de sus planteles el } \\
\text { trabajo con proyectos formati- } \\
\text { vos integradores como estrate- } \\
\text { gia didáctica que le permita al } \\
\text { estudiante el desarrollo en su } \\
\text { contexto, abordando problemas } \\
\text { de proponiendo alternativas de } \\
\text { solución. COBAEH tiene expe- } \\
\text { riencia en este tipo de trabajo, } \\
\text { convertirse alternativa anima- } \\
\text { ción sociocultural, es decir en } \\
\text { una practica contextualizada de } \\
\text { interacción social, Tomando en } \\
\text { consideración las ventajas que } \\
\text { ofrece la estrategia como: } \\
\text { Desarrollo de habilidades y } \\
\text { considerando puede éste en una } \\
\text { para la competencias colabora- } \\
\text { tivo. Trabajo que los estudian- } \\
\text { tes se educan en colectivo y que } \\
\text { la educación se basa en comu- } \\
\text { nicación, justamente formativo } \\
\text { integrador que permita el inte- } \\
\text { rés de los estudiantes en el } \\
\text { ámbito local, principalmente } \\
\text { porque no existe un espacio } \\
\text { físico para la interacción social }\end{array}$ & $\begin{array}{l}\text { "Consiste en un ejercicio inves- } \\
\text { tigativo que los estudiantes } \\
\text { desarrollan en cada semestre } \\
\text { académico en el cual deben } \\
\text { articular todos los saberes que } \\
\text { cursan para responder al núcleo } \\
\text { problemático de su nivel de } \\
\text { formación. Por ser una estrate- } \\
\text { gia de investigación formativa } \\
\text { no se orienta a la generación de } \\
\text { un conocimiento objetivamente } \\
\text { nuevo sino a la apropiación } \\
\text { comprensiva de conocimientos } \\
\text { ya elaborados pero nuevos para } \\
\text { el estudiante. A través de esta } \\
\text { estrategia se pretende el fortale- } \\
\text { cimiento de los aprendizajes } \\
\text { significativos." } \\
\text { "La elaboración del proyecto } \\
\text { integrador implica que los estu- } \\
\text { diantes al cur- sar cada uno de } \\
\text { sus semestres académicos deli- } \\
\text { miten una problemática y visua- } \\
\text { licen su solución con los recur- } \\
\text { sos científicos disponibles. La } \\
\text { integración del conocimiento se } \\
\text { logra a partir del diseño y ejecu- } \\
\text { ción de un ejercicio de investi- } \\
\text { gación que se articula con la } \\
\text { intervención en el con- texto en }\end{array}$ \\
\hline
\end{tabular}


HERNÁNDEZ: IDENTIDAD NACIONAL COMO PUNTO...

\begin{tabular}{|l|l|l|l|}
\hline Concepto & Clasificación & \multicolumn{1}{|c|}{ Justificación social } & \multicolumn{1}{|c|}{ Marco Teórico } \\
\hline & & como centro comercial, cine, & el que participan”. Nohelia H. \\
& & una infraestructura en materia & $(2007: 236-238)$ \\
& de cultura. Metodología de & \\
& intervención, prevención de & \\
& problemas sociales y creación & \\
& de ambientes de convivencia e & \\
& interacción social, promoción & \\
& cultural, artística y deportiva. & \\
& Promueve el desarrollo de los & \\
& jóvenes que construyen vínculos & \\
& para provocar transformaciones. & \\
\hline
\end{tabular}

Fuente: Elaboración propia con datos de trabajo colegiado en el COBAEH Tizayuca, 2014.

La matriz anterior, nos permite, de manera general, comprender cómo se va desarrollar la matriz de encuentro y alineamiento de las universalidades y especificidades. Este sistema servirá como base para el debate en los Foros Educativos, así llegar de manera local-global a acuerdos que permitan un rescate paulatino de la identidad nacional. Teniendo a la estructura de la EMS como principal herramienta y alternativa de solución. La participación de las instituciones educativas de Educación Superior de Hidalgo es primordial para el desarrollo de este diálogo académico. Es el caso de la máxima casa de estudios del Estado de Hidalgo, la Universidad Autónoma del Estado de Hidalgo, mediante los especialistas, investigadores y docentes deben formar parte de los encuentros educativos (Foros de Consulta Nacional para la Revisión del Modelo Educativo) para discutir y ensamblar los contenidos universales y específicos al siguiente peldaño educativo para los alumnos.

\section{Reflexiones finales}

La Educación Media Superior ha sufrido grandes transformaciones con miras a mejoras pertinentes y permanentes. Es necesario utilizar estas plataformas educativas como herramientas promotoras de estabilidad social.

La modernidad, como la hemos planteado, ha tornado de rasgos efímeros a las estructuras sociales, desde los modos de comunicación, hasta lo que aquí nos ha preocupado, el sistema de valores. Los valores, se entienden como ese conjunto de ideas que mantienen cohesionados a integrantes de grupos heterogéneos. Es por eso la importancia de la resignificación planteada.

Los espacios están creados, se ponen en marcha, sólo es aprovechar al máximo las reuniones planeadas por las autoridades educativas. En este caso, los Foros de Consulta Nacional para la Revisión del Modelo Educativo servirán de plataforma para el desarrollo de proyectos que proyecten un rescate de la tranquilidad social.

Asimismo, para la integración de especialistas del nivel superior, las autoridades nacionales de la Educación Media Superior, deben buscar la contribución de instituciones educativas de prestigio y calidad. El ejemplo claro de lo anterior, la Universidad Autónoma del Estado de Hidalgo contribuye en el campo de la ciencia social, mediante el Instituto de Ciencias Sociales y Humanidades, por lo tanto, formar un lazo de cooperación académica en los Foros de Consulta Nacional para la Revisión del Modelo Educativo es primordial para el acercamiento al funcionamiento ideal de la presente propuesta.

Es importante comprender que la característica de fluidez que se le otorga al sistema de valores actual, es difícil de combatir y que se deben crear muchas estrategias para mitigar las consecuencias de la modernidad. Centrarse en la resignificación, como planteamiento del rescate de puntos de encuentro entre las comprobadas heterogeneidades (multiculturalismo) nos permite suponer un eje de acción de solidificación de la estructura de valores. 


\section{REFERENCIAS}

Amérigo, M. (2006). La investigación en España sobre actitudes pro- ambientales y comportamiento ecológico. Medio Ambiente y Comportamiento Humano, 7(2), pp. 45-71.

Bauman, Z. (2002). La cultura como praxis. Barcelona: Paidós.

- (2007). Tiempos líquidos. Barcelona: Tusquets.

Beck, U. (1998). ¿Qué es la globalización? Falacias del globalismo, respuestas a la globalización. Barcelona: Paidós.

Casas, R. M. (2007). Vivir invisibles: la resignificación cultural entre los otomíes urbanos de Guadalajara. Ciesas. Recuperado en: http://books.google.es/books?hl=es\&lr=\&id=dnXsIb6PA $\mathrm{X} 4 \mathrm{C} \&$ oi $=$ fnd\&pg=PA15\&dq=resignificación\&ots $=$ SceDokxDWo\&sig=n1hM0pflcbZPTnHv3PdtiqKDSk\#v=onepage \&q=resignificación\& $\mathrm{f}=$ false

Castells, M. (1995). La ciudad informacional: tecnologías de la información, reestructuración económica y el proceso urbano- regional. Madrid: Alianza Editorial.

- (2000). La era de la información. Tomos I, II y III. México: Siglo XXI editores.

Castoriadis, C. (1998). The Imaginary Institution of Society. Cambridge: The MIT Press.

Consejo Nacional de Evaluación de la Política de Desarrollo Social. (2008). Metodología para la medición multidimensional de la pobreza en México. CONEVAL. Consultado el 30 de agosto de 2012 en: http://bit.ly/LEUriz

- (2012). Diagnóstico del avance en monitoreo y evaluación en las entidades federativas 2011. México, D.F. CONEVAL. Consultado el 30 de agosto de 2012 en: http://ow.ly/dFcMs

- (2012). Inventario CONEVAL de Programas y Acciones Estatales para el Desarrollo Social 2010. CONEVAL. Consultado el 10 de octubre de 2012 en: http://web.coneval.gob.mx/Evaluacion/IPE/Paginas/default.aspx

- (2012). Sección de Entidades Federativas. CONEVAL. Consultado el 30 de agosto de 2012 en: http://www.coneval.gob.mx/

Delors, J. (1996). La educación encierra un tesoro. Informe a la UNESCO de la Comisión Internacional sobre la educación para el S. XXI. Madrid: Santillana-UNESCO.

Diario Oficial de la Federación. (2004). Ley General de Desarrollo Social, 20 de enero de 2004. México. Consultado el 30 de agosto de 2012 en: http://www.diputados.gob.mx/LeyesBiblio/pdf/264.pdf.

- (2005). DECRETO por el que se regula el Consejo Nacional de Evaluación de la Política de Desarrollo Social, 24 de agosto de 2005. México. Consultado el 30 de agosto de 2012 en: http://www.coneval.gob.mx/cmsconeval/rw/resource/coneval/normateca/234 3.pdf?view $=$ true

Frías, N., Ibarra, M. y Rivera, L. (2006). La organización comunitaria. Actor ausente en la reglamentación del Programa 3x1 para migrantes en Hidalgo. En R. Fernández, R. García y A. Vila (coords.), El Programa 3xl para migrantes. ¿Primera política trasnacional en México? (pp. 160-210). México: Instituto Tecnológico Autónomo de México/ Universidad Autónoma de Zacatecas / Miguel Ángel Porrua.

González, A. y Amérigox, M. (1999). Actitudes hacia el medio ambiente y conducta ecológica. Psicothema, 11(1), pp. 13-25.

Gutmann, AMY. (1987). Democratic Education. Princeton: Princeton University Press.

Ramirez, N. H. (2007). El Proyecto Integrador: Una estrategia pedagógica para lograr la integración y la socialización del conocimiento. Bogotá: Universidad de San Buenaventura.

Tuirán, R. (2002). Migración, remesas y desarrollo. Boletín migración internacional, 6(19). 


\section{SOBRE EL AUTOR}

Fabián Hernández Galicia: Docente de la Universidad Autónoma del Estado de Hidalgo, México. Docente del Colegio de Bachilleres del Estado de Hidalgo, México. Docente Certificado a Nivel Nacional (Sistema basado en Competencias). Licenciado en Sociología. Pasante de la Maestría en Tecnología Educativa. 